\title{
National Standardization Activities Within China
}

\author{
Zhiyong Feng, Ping Zhang and Ying $\mathrm{Xu}$ \\ Key Laboratory of Universal Wireless Communications, Ministry of Education \\ Wireless Technology Innovation Institute (WTI) \\ Beijing University of Posts and Telecommunications, Beijing, P.R.China, 100876 \\ Email: \{fengzy,pzhang\}@bupt.edu.cn, xuying.bupt@gmail.com
}

\begin{abstract}
Cognitive Radio (CR) is an efficient method to improve the spectrum efficiency. Industry and academic fields have focused on CR in recent years. Plenty of works on standardization of $C R$ are concentrated among different standard organizations such as IEEE and ETSI, etc. This paper mainly introduces the work on standardization of $C R$ in China. The proposals submitted to ITU-R from China is also introduced. Then the paper discusses the application scenarios of $C R$ and overviews the standardization progresses of CR in CCSA. Finally, the testbed of CRS in China is discussed.
\end{abstract}

\section{INTRODUCTION}

The explosive growth of capacity-hungry wireless services and rapid development of wireless technology are presenting important challenges to the scarce spectrum resource, and the actual reason is inefficient spectrum utilization due to static spectrum allocation policy in [1]. Cognitive Radio (CR) is presented by Joseph Mitola III and Gerald Q. Maguire, Jr. [2] to capture the challenge on how to efficiently utilize spectrum resource.

$\mathrm{CR}$ is considered as the concept with the context-aware intelligent radio technology which has the potential capability to complete the reconfiguration autonomously by learning from the changing environment [3]. Due to the efficient utilization of spectrum, CR has attracted many research studies and discussion from plenty of countries and organizations. Companies like Vanu, Inc. and Shared Spectrum Company, projects like the DARPA XG, the EU E2R and E3, the Dutch Adaptive Ad Hoc Free Band Wireless Communications (AAF) project, and research groups in numerous universities are focusing on CR [4]. The research from Vanu, Inc. and Shared Spectrum Company is oriented toward the needs of civil wide area (cellular) and short-range communication systems, as demonstrated by early prototypes of institutions. The EU E2R and E3 give more focus on ability of reconfiguration by CR [5]. Following with these projects, many standard organizations work on a series of standards on CR. Several CR standards are currently under development, mainly within the IEEE and the European Telecommunications Standards Institute (ETSI) framework. IEEE 802.22, which is created in 2004, has developed a standard for wireless regional area networks (WRANs) using white spaces in the TV frequency spectrum. Other IEEE 802 Working Groups (WGs) such as 802.11 af and 802.11y, etc., work on Cognitive Radio Networks (CRNs) within TV band. IEEE SCC41 is developing standards related to dynamic spectrum access networks with a focus on improved spectrum usage. Further standards are currently in preparation within IEEE SCC41, including P1900.4a for enabling mobile terminals to access the white space frequency bands without any limitation on the radio access technology. CR principles within ETSI RRS are concentrated on two topics, cognitive pilot channel proposal and functional architecture for management and control of reconfigurable radio systems, including dynamic self-organizing planning, dynamic spectrum management, joint radio resource management, etc [5].

Since many countries and organizations are working on standards for $\mathrm{CR}$, and the technical, managerial, and financial aspects are associated with CR concepts, there is a need to standardize processes, terms, and so on. ITU-R set up work groups (WGs) on CR responsible for CR definition and rules of the Radio Regulations for CR. Cognitive radio system (CRS): A radio system employing technology that allows the system to obtain knowledge of its operational and geographical environment, established policies and its internal state; to dynamically and autonomously adjust its operational parameters and protocols according to its obtained knowledge in order to achieve predefined objectives; and to learn from the results obtained[6]. The illustration of Cognitive Radio System (CRS) concept from ITU-R is as below Fig.1. ITU-R 5A is working on definition and radio rule of CRS; and ITU-R 5D is working on how to combine CRS concept with IMT-Advanced system. Plenty of countries and organizations have submitted many proposals about CRS. China has also contributed a lot respectively on obtaining knowledge, learning and decision making. These proposals are from research within industry and academic fields. China has developed many projects on CR such as National Basic Research Program, Key Project of National Natural Science Foundation, and National Key Technology RD Program, etc. The standard organization in China, China Communications Standards Association (CCSA), has organized proposals from industry and academic fields to submit to ITU-R.

This paper introduces the development of standardization on CR in China. The proposals are overviewed from ITU-R CRS definition with obtaining knowledge, learning and decision making. Then the application scenarios are analyzed, the standard progress in CCSA is introduced. Finally, the testbed of CRS in China is overviewed and the future work on activities of CR standardization in China is expected. The rest of paper is organized as follows. Section II introduces the proposals to 


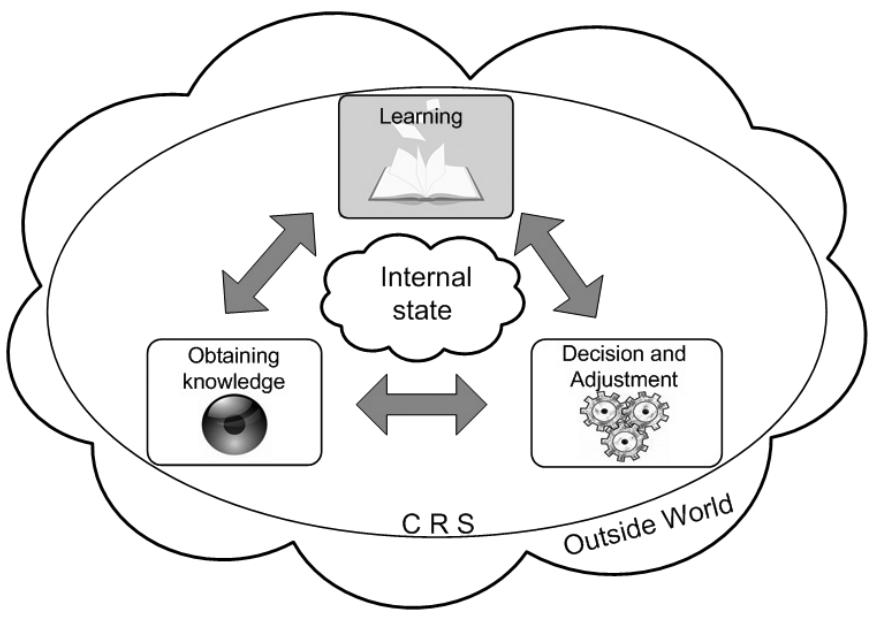

Fig. 1. Illustration of CRS for ITU-R 5A [6]

ITU-R from China. Section III overviews the progress of the standards process in CCSA. Section IV introduces testbed of CRS in China. Finally, Section V concludes the paper.

\section{Proposal ON CR IN ITU-R AND ChinA}

\section{A. Technical Proposal on CR in ITU-R and China}

In this section, we give an introduction about the technical characteristics and capabilities of CRS defined in ITU and the contributions from China.

The three key technical functions characterize a CRS defined in ITU are [7]:

- Obtaining knowledge: The ability to obtain the knowledge about its surrounding radio operational and geographical environments, its internal state, the spectrum policies, and users' specific needs, etc.

- Decision making and adjustment of operational parameters and protocols: The ability to dynamically and autonomously execute optimization processes, e.g., adjust operational parameters and protocols, to achieve predefined goals, based on the obtained knowledge.

- Learning: The ability to learn from the feedback of its optimization actions, to further improve the performance of the later decision making.

1) Obtaining Knowledge: Generally, the knowledge can be divided into two types, that is,

- The knowledge of elements and status internal to the CRS, including, configuration of the CRS (e.g., spectrum bands used by the CRS and coverage area of the CRS), status of nodes of the CRS (e.g., positions, load, interference level), etc.

- The knowledge of elements and status external to the CRS, including, current usage of specific spectrum bands, positions of other base stations, interference levels at the other base stations, spectrum policies, users' needs and distribution of them in the geographic area of CRS.

Then, a corresponding question arises, that is how to obtain the aforementioned knowledge. In [7], five basic methods are given, i.e., collected information by CRS nodes, listening to a wireless control channel, spectrum sensing, database access, Geo-location, and collaboration between different radio systems. Specifically, the collaboration method is proposed by China [8], which means the CRS may obtain knowledge from other systems by collaboratively exchanging their information, which may includes the information of their Geo-location, channel state information, etc. In the following, we present the key contributions from China.

For spectrum sensing method, the waveform-based detection method is proposed [7]. This method is suitable for the case when known patterns of other wireless systems, e.g., preambles, mid-ambles and spreading codes, are available to CRS. Specific patterns are usually used in wireless system to assist some key process, e.g., synchronization. Compared to other sensing methods, the sensing time of waveformbased method is shorter and the performance increases as the length of the known signal pattern increases. Additionally, performance indicators in spectrum sensing are also proposed to be taken into account of [9], e.g., algorithm complexity, detection probability and false alarm probability, etc.

For database access method, the concept of multi-dimension cognitive database is proposed [10]. According to fact that the cognitive information in CRS is comprehensive, e.g. information on space, time, and frequency, the cognitive database should contain several dimensions of cognitive information in terms of the nature of the cognitive information, e.g., radio dimension, network dimension, user dimension and policy dimension.

For Geo-location method, a mesh-based approach for organization of geographical related information for cognitive pilot channel (CPC) is proposed [11]. For brevity, interested readers can refer to [7] for details about CPC. In the proposal, the CPC operates in a certain geographical area that can be virtually divided into meshes. A mesh refers to a region where certain radio electrical commonalities can be identified, e.g., power above a certain level is detected at a certain frequency in the coverage area of the mesh. The coverage area of different networks can be divided into a set of meshes in a certain geographical area. CPC delivers cognitive information based on mesh division, where each mesh has different operational state, such as traffic load and radio access technology. There are mainly three information delivery methods: broadcast mode, on demand mode and multicast mode. In particular, the multicast mode is proposed [11], where the network would wait the requests from users in the same mesh for a period time before sending the information into the scheduling system.

2) Decision Making and Adjustment of Operational Parameters and Protocols: The second key characteristic of the CRS is the ability to analyze the obtained knowledge and dynamically and autonomously make decisions on the reconfiguration. The decisions can be made in a centralized or distributed manner. After the decisions on the reconfiguration have been made, the CRS do the correspondingly reconfiguration according to the decisions. There are four methods of making decisions and adjustments, that is, centralized decision 
making, distributed decision making, method of adjustment based on software defined radio (SDR) reconfiguration and method of adjustment based on CRS reconfiguration. Here we give a brief introduction about centralized decision making proposed by China [7] as an example, and for brevity, interested readers can refer to [7] for details about the other three methods of making decisions and adjustments. The centralized decision making is suitable for scenarios where a central node is deployed. The central node collects the information from all nodes of CRS and makes a global optimization decision. The centralized way is simple and easy to control from the perspective of network operators. On the other hand, the complexity would increase rapidly with the increase of CRS nodes correspondingly.

3) Learning: The third key characteristic of the CRS is the ability to learn, the aim of which is to improve the performance of the decision making process by using previous actions and results. Many machine learning algorithms can be used in CRS, among which, game theory and policy engines are the top two popular techniques under investigation for CRS management. According to available cognitive information, CRS may improve the existing learning algorithms. The outcome of the learning process is used to build a knowledge basis for future transmissions.

\section{B. Application Scenarios on CR in China}

Deployment scenarios which illustrate application of CRS technology for both single operator and multiple operators are introduced here. While for IMT systems, the use of CRS technology by an operator of radio communication systems to improve the management of assigned spectrum resource is focused.

1) Guide terminal reconfiguration: In this scenario, the area is overlapped by multiple Radio Access Networks (RANs) which employ different Radio Access Technologies (RATs) on different frequencies. Two possible examples of this scenario are identified by ITU. These two examples are characterized by different capability of terminals. In the first example, some of the terminals are required to be reconfigurable. While in the second example, the terminals are more likely to be multimode ones.

In the first example, some of the terminals are reconfigurable that means, they can adjust their operational parameters and protocols to use different RATs. Such terminals can obtain knowledge required for decision making and dynamically implement such adjustment. Also, radio systems may assist terminals in obtaining knowledge and guide terminals in their reconfiguration decisions. Cognitive pilot Channel (CPC) and multi-dimension database are candidate solutions for heterogeneous network information delivery.

In the other example, some terminals have the capability to communicate with the different radio systems, but they are not reconfigurable. In this case, additional multi-RAT stations with reconfigurable capability can be deployed to serve as a bridge between current existing radio systems and terminals. Such multi-RAT stations obtaining knowledge about the operational environment, and adjust their operational parameters and protocols to connect to current existing radio systems while providing connection to terminals using one RAT.

2) Improve network management: With the development of Dynamic Spectrum Management (DSM) and Joint Radio Resource Management (JRRM), network management can be easily done by introducing CR technology to current radio systems. Strong leaning ability is required and emphasized here.

\section{- Single operator}

In this scenario, an operator usually owns several networks covering the same geographical area. It is wellknown that in a certain geographical area, the patterns of traffic may be non uniform in time and in space. In some area, this may leads to a situation that in some networks the traffic is heavier or even overloaded while in the other networks the load is extremely light. Exploiting CRS capabilities, the operator owning two or more RATs could dynamically and jointly manage the resources of the deployed RATs in order to make the network adapted to the dynamic traffic behavior and to globally maximize the capacity.

- Multiple operators

The CRS technologies may enable cooperation between networks owned by different operators to mitigate the interference and congestion issues. Exchange information among networks on the usage of their respective assigned spectrum may avoid harmful interference between different systems. Interference problems may occur between networks and private networks, as well as, between multiple private networks due to the possible usage of the same spectrum bands and the interaction between macro-cells and femto-cells. CRS technologies allow users to detect vacant spectrum and intelligently choose a spectrum band to operate on without causing much interference.

Exchanging information among networks on the usage of their respective assigned spectrum allows operators to share this spectrum. The variations in the occupancy of the assigned spectrum in a specific location at a specific time enable the improvement of spectrum efficiency by taking advantage of parts of the unused spectrum resulting from these variations. The capability to predict these variations in advance is one of the problems to deal with. In addition, traffic load-balancing between the public land mobile networks and private networks is another issue. Additionally, people have not jointly considered the technical and the economic issues, which are essential for applying DSM in real systems, especially for multioperator scenarios.

3) Opportunistic spectrum access: In this scenario there is no priority determination of the spectrum to be eventually accessed by the interested party. CRS access currently unused spectrum and services without causing harmful interference. The key issue to be addressed is the selection of the spectrum 
that can be eventually accessed.

There are three kind of selection method considered by ITU. A common method is that CRS identify the unused spectrum based on a real-time radio environment analysis. When occupancy of the spectrum does not significantly vary over time, unused parts of the spectrum are available in a more static manner. So CRS seize this character of network systems, and choose the stable unused spectrum. Database is the third method when systems share bands in a horizontal manner.

4) Capacity or coverage extension: According to the dynamic change of both user's behavior and radio characteristics, network planning leaves coverage holes as well as areas lacking capacity in many cases. This problem can be solved by deploying additional base stations using CRS technology managed by the same operator or by new entrant operators, when allowed by regulator department. For hot spot, this additional deployment could increase the capacity of current system so that the congestion problem can be mitigated. The frequency band used by this added base station is lent from the unused spectrum bands of other systems. For blind area, such added base station may use the same frequency band that networks may suffer from mutual interference.

\section{KPI on spectrum sensing for CR Technology}

CRS is designed to improve the system performance without introducing extra interference to current systems. But, the evaluation of the increase in performance and the cost of this increase are still to be addressed. And key performance indicator in spectrum sensing is discussed by ITU.

$\mathrm{CR}$ is designed to be aware of and sensitive to the changes of its surrounding, which makes spectrum sensing an important requirement for the realization of cognitive radio networks. Currently there are a series of spectrum sensing methods investigated for cognitive radio systems, including matched filter detection, energy detection, and feature detection etc. These existing sensing methods differ in their sensing performances, and are suitable to be implemented in various circumstances. Therefore it is necessary to define key performance indicators of spectrum sensing to evaluate the performance of different methods.

- Detection threshold for the signals of the existing system. This is minimal signal-to-noise ratio (SNR) which is needed by each spectrum sensing method in various existing systems to achieve a certain probability of detection. Detection time for the signals of the existing system. This is the duration which is used by each spectrum sensing method to detect the signals of existing system.

- Algorithm complexity: The complexity of each spectrum sensing method related to the observed bandwidth.

- Detection probability: Probability that the signal is correctly detected when it is present.

- False alarm probability: This refers to the probability that the signal is detected when it is not present.

- Sensing signaling cost: Sensing signaling cost generally includes cost in sensing measurement and sensing report.
For cooperative sensing, signaling cost in sensing report combining should also be included.

- Time between failures in detection: Average time period between failures in signal detection (i.e. signal is not detected when it is present).

- The lost spectrum opportunity ratio: This refers to the expected fraction of the OFF state (i.e., idle time) undetected by CR users.

- The interference ratio: This refers to the expected fraction of the ON state (i.e., the transmission time of the networks of the existing systems) interrupted by the transmission of CR users.

\section{Standard Progress in CCSA}

In December of 2008, during the 27th meeting of TC5 WG8 of CCSA, the proposal of draft a report on CR dealing with WRC-12 Agenda item 1.19 was accepted, and this report will be finished at the end of 2010 according to the initial plan.

This report includes three parts: part one will analyzes the technologies of CRS, part two will introduce the standard progress in ITU, IEEE and so on, and part three will analyze the possible impact on spectrum management.

The first part will analyze the technologies of CRS, such as the concept of CRS, the characteristic of key technologies, the possible application of CRS, including applications in IMT system, and applications in White Space, and the performance evaluation of CRS related technologies.

The second part will introduce the standard progress in ITU-R(5A, 5D and 1B), ETSI, IEEE and SDR forum, the developing trends of CRS. The third part will analyze the possible influence on spectrum management considering the usage of CRS technology. Firstly, the requirement of CRS will be introduced; secondly, the possible spectrum management problem caused by CRS will be analyzed; then the possible spectrum management methods for CRS will be proposed; at last, the spectrum management policy for CRS will be discussed, including from legal aspect, from administrative aspect, from economy aspect and from technical aspect.

\section{Testbed OF CRS IN CHINA}

In order to verify some key technologies of CRS, to advance the standardization of CRS, and also in order to promote TD-LTE systems development in China, a TD-LTE oriented cognitive wireless networks testbed is being developed in China.

The purpose of this testbed is to verify the heterogeneous network convergence and spectrum usage efficiency improvement. The working band of this testbed is from $2.3 \mathrm{GHz}$ to $2.4 \mathrm{GHz}$ which is the shared-band of IMT-A and Radar system. The primary User is Radar, and the cognitive user is TD-LTE system. To add functions related to cognition capability the protocols of TD-LTE system has been modified based on 3GPP 36 series R8 accordingly. The architecture of this testbed is show in Fig. 2 .

Classified by function entities, the platform is mainly made of part of the network side, the base stations, terminals and other components. 


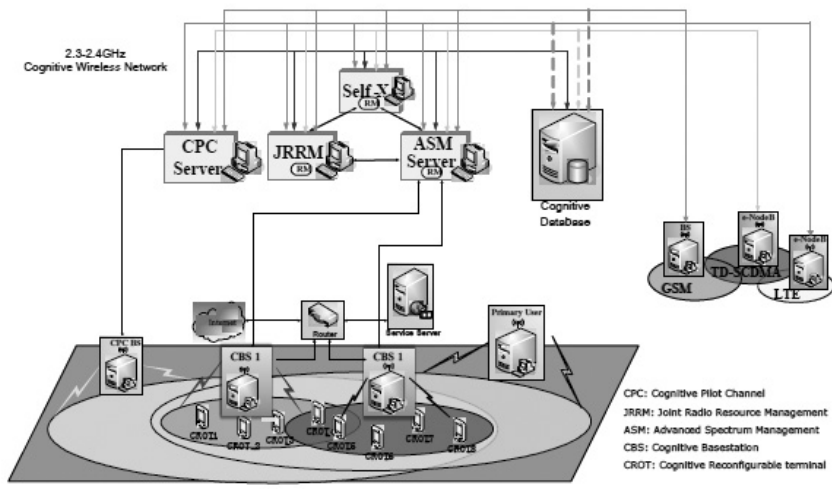

Fig. 2. Architecture of the CRS testbed

For the network side, there are some entities. such as CPC Server, JRRM Server, ASM (Advanced Spectrum Management), and Cognitive Database.

There are two cognitive base stations and eight cognitive terminals working in TD-LTE mode in this testbed to support the dynamic spectrum access. The cognitive base station has the capability of spectrum sensing without quiet period and the capability of dynamic usage of spectrum, and the cognitive terminals don't need to do spectrum sensing, but they can adjust themselves according to the change of the outside environment. Besides these, this testbed can also works in heterogeneous radio access networks environment, including TD-LTE, TD-SCDMA, GSM / GPRS network such as the seamless integration of heterogeneous target networks.

The deployment scenarios of this testbed include Dynamic Spectrum Access of TD-LTE system and joint Radio Resource Management of heterogeneous radio networks.

Besides this testbed, the testbed of TD-LTE in white space based on CR technologies is also being considered in China and will be planned in the near future.

\section{CONCLUSION}

This paper introduces the development of CR standard ITU$\mathrm{R}$ from China's perspective. The technology and application scenarios on CR standards in ITU-R and China are analyzed. The progress in CCSA is overviewed, and the testbed of CRS in China is introduced. There should be a long road for CR development considering some technologies of CRS are still not mature, so further research and testbed or field trial on CR will push forward the improvement of CRS.

\section{ACKNOWLEDGMENT}

This work was sponsored by National Basic Research Program of China (973 Program) (2009CB320400), National Key Technology RD Program of China (2010ZX03003-00101,2009ZX03007-004) and Key Project of National Natural Science Foundation of China (60832009). The contributions of the colleagues from National Basic Research Program of China (973 Program) are hereby acknowledged. The author would also like to extend great gratitude to the colleagues from
Wireless Technology Innovation Institute of Beijing University of Posts and Telecommunications.

\section{REFERENCES}

[1] FCC Spectrum Policy Task Force, "Report of the spectrum efficiency working group," Nov. 2002. [Online]. Available: http://www.fcc.gov/sptf/reports.html

[2] J. Mitola III and G. Q. Maguire Jr., "Cognitive Radio: Making Software Radios More Personal," IEEE Pers.Commun., vol. 6, no. 4, Aug. 1999, pp. 13-18.

[3] J. Mitola, "Cognitive radio," Licentiate proposal, KTH, Stockholm, Sweden.

[4] Prasad, R.V, Pawelczak, P, Hoffmeyer, J.A, Berger, H.S, "Cognitive functionality in next generation wireless networks: standardization efforts", IEEE Communications Magazine, vol.46, no.4, pp.72-79, April 2008.

[5] Mueck, M. Piipponen, K. Dimitrakopoulos, G. Tsagkaris, K. Demestichas, P. Casadevall, etc, A. ’ETSI reconfigurable radio systems: status and future directions on software defined radio and cognitive radio standards", IEEE Communications Magazine, vol.48, no.9, pp.78-87, Sep 2010.

[6] Question ITU-R 241-1/5, "Annex 12 to Working Party 5A Chairman's Report-Cognitive radio systems in the land mobile service", Document 5A/TEMP/268

[7] ITU-R, "Cognitive radio systems in the land mobile service", Document5A/TEMP/601-E, Working Party 5A, 22 November 2010.

[8] ITU-R, "Proposal for Section 5 of Working Document Towards a Preliminary Draft New Report ITU-R [LMS.CRS]", Document 5A/TEMP/469E, Working Party 5A, 30 April 2010.

[9] ITU-R, "Key Performance Indicators for Spectrum Sensing", Document 5A/TEMP/376-E, Working Party 5A, 18 November 2009.

[10] ITU-R, "Database for Cognitive Radio System", Document 5A/TEMP/377-E, Working Party 5A, 18 November 2009.

[11] ITU-R, "Multicast CPC Mode in Cognitive Radio Environment", Document 5A/TEMP/375-E, Working Party 5A, 18 November 2009. 